\title{
Caracterização estrutural e potencial florestal para o manejo comunitário da Floresta Nacional do Purus, Amazônia Ocidental
}

\author{
Structural characterization and forest potential for community management of \\ Purus National Forest, Western Amazonia
}

\author{
Pedro Christo Brandão', Agostinho Lopes Souza ${ }^{\mathrm{II}}$, \\ Alexandre Quinet ${ }^{\mathrm{III}}$, Bruno Araujo Furtado de Mendonça ${ }^{\mathrm{IV}}$
}

\section{Resumo}

\begin{abstract}
A Floresta Nacional (FLONA) do Purus, situada no município de Pauiní-AM, apresenta elevado estado de conservação e uma população de aproximadamente mil habitantes. No entanto, a carência de estudos ambientais em escala adequada para o planejamento da gestão da FLONA torna imprescindíveis levantamentos que quantifiquem e qualifiquem o estoque de recursos, identifiquem a demanda comunitária e fundamentem técnicas de produção florestal sustentável. Nesse sentido, o objetivo do presente trabalho foi analisar a composição florística, a estrutura florestal e o potencial de manejo florestal de um trecho de floresta ombrófila densa de terra firme, localizado na Unidade de Manejo Florestal-1 (UMF-1) da Vila Céu do Mapiá, maior núcleo populacional da FLONA. Para tanto, foi realizado um inventário florestal por amostragem aleatória de 12 parcelas de $50 \times 50 \mathrm{~m}$, sendo mensurados todos os indivíduos com DAP $\geq 10 \mathrm{~cm}$. Foi realizada a análise da composição florística, bem como utilizados os índices de diversidade Shannon-Wienere de equabilidade de Pielou, para avaliar a diversidade e uniformidade da comunidade. A análise da estrutura da floresta foi baseada nos valores absolutos e relativos de densidade, dominância, frequência, valor de importância e distribuição diamétrica, da área basal e estimativas volumétricas do povoamento. Foram registrados 1.764 indivíduos, pertencentes a 35 famílias botânicas, 91 gêneros, e 163 possíveis espécimes, ressaltando que menos de $40 \%$ (65) foram identificadas em nível de espécie. A UMF1 caracteriza-se pela alta diversidade florística acumulada da comunidade arbórea amostral $\left(\mathrm{H}^{\prime}=4,10\right)$, poucas espécies dominantes $(J=0,81)$ e muitas espécies de baixa abundância. Das espécies comerciais utilizadas na comunidade, três figuram entre as dez espécies com maior valor de importância, em um total de 26 espécies comerciais possíveis de exploração sustentável.
\end{abstract}

Palavras-chave: Composição florística; Inventário florestal; Identificação botânica; Espécies madeireiras

\footnotetext{
Engenheiro Florestal, Dr., Instituto Socioambiental de Viçosa, Sítio Palmital, s/n, CEP 36578-899, Viçosa (MG), Brasil. pedrocbrandao@gmail.com (ORCID: 0000-0003-3937-9022)

Engenheiro Florestal, Dr., Professor Aposentado do Departamento de Engenharia Florestal, Centro de Ciências Agrárias, Universidade Federal de Viçosa, R. Purdue, CEP 36570-900, Viçosa (MG), Brasil. alsouzaal@gmail.com (ORCID: 0000-0003-0205-2392)

III Biólogo, Dr., Pesquisador do Instituto de Pesquisa Jardim Botânico do Rio de Janeiro, Unidade de Botânica Sistemática, Rua Pacheco Leão 915, CEP 22460-030, Rio de Janeiro (RJ), Brasil. aquinet@jbrj.gov.br (ORCID: 0000-0001-9749-9554)

IV Engenheiro Florestal, Dr., Professor do Departamento de Silvicultura, Instituto de Florestas, Universidade Federal Rural do Rio de Janeiro, BR 465 km 07, CEP 23890-000, Seropédica (RJ), Brasil. brunoafmendonca@gmail.com (ORCID: 0000-0003-0288-0024)
} 


\begin{abstract}
Purus National Forest (PNF), located in the municipality of Pauiní, Amazonas state, Brazil, has a high conservation status and a population of about one thousand inhabitants. However, the scarcity of environmental studies in a suitable scale for the PNF management planning demands studies that quantify and qualify the stock of resources, that identify the community demand and set sustainable forest production techniques. Thus, the objective of the present work was to analyze the floristic composition, forest structure and the forest management potential of part of a "terra firme" dense ombrophylous forest located in the Forest Management Unit (UMF-1) of Céu do Mapiá village, the largest populational nucleus of PNF. To do so, a forest inventory was made, sampling in a block of 12 parcels of 50x50m, and all trees with $\mathrm{DBH} \geq 10 \mathrm{~cm}$ were measured. The floristic composition was carried out and the Shannon-Wiener diversity index and Pielou equability index were used to assess the biodiversity and the uniformity of the community. The structural forest analysis was based on the absolute and relative values of density, dominance, frequency, importance value and diameter distribution of the basal areas and volumetric estimates of the population. A total of 1,764 individuals, pertaining to 35 botanic families, 91 genera and 163 possible specimens were registered, emphasizing that less than $40 \%(65)$ were identified at the species level. The UMF-1 is characterized by the high floristic diversity $\left(\mathrm{H}^{\prime}=4.10\right)$, few dominant species $(\mathrm{J}=0.81)$ and many species with low abundance. Out of the commercial species used by the community, three are among the ten species with the greatest importance value, a total of 26 possible commercial species of sustainable exploitation.
\end{abstract}

Keywords: Floristic composition; Forest inventory; Botanical identification; Timber species

\title{
Introdução
}

Em razão de uma série de aspectos políticos, econômicos e climáticos, a Floresta Amazônica está sendo destruída-desflorestada em ritmo acelerado (SPRACKLEN; GARCIA-CARRERAS, 2015; SONTER et al., 2017; ARAGÃO et al., 2018). Só nos últimos 25 anos foram devastados 6\%

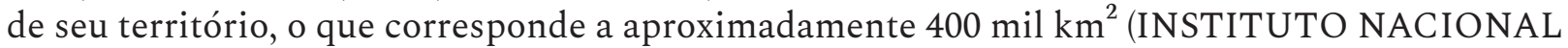
DE PESQUISAS ESPACIAIS, 2013). Apesar desse cenário de destruição, algumas iniciativas estão sendo tomadas pelo Poder Público e sociedade civil na busca de um novo paradigma de desenvolvimento para a região. O estabelecimento de uma legislação ambiental modelo, pautada na aplicação de critérios de sustentabilidade do manejo florestal (BRASIL, 2009) e a criação de mosaicos de áreas protegidas (Unidades de Conservação e Terras Indígenas) são medidas que estão sendo implementadas nesse sentido.

A Floresta Nacional do Purus (FLONA do Purus), criada pelo Decreto Federal n. ${ }^{\circ} 96.190$ de 21/06/88 (BRASIL, 1988), é uma Unidade de Conservação de Uso Sustentável, situada no município de Pauiní, Estado do Amazonas, que tem por objetivo promover a pesquisa e manejo florestal sustentável aliados à conservação que compreende uma área de floresta natural de aproximadamente 256 mil hectares. A FLONA possui aproximadamente 1.000 habitantes e apresenta elevado estado de conservação e potencial para o ecoturismo e para o manejo florestal sustentável comunitário de produtos florestais madeireiros e não madeireiros (INSTITUTO CHICO MENDES DE CONSERVAÇÃO DA BIODIVERSIDADE, 2009). No entanto, não há estudos detalhados suficientes para o planejamento da gestão da FLONA do Purus, especialmente relacionados ao potencial de manejo florestal sustentado para uso múltiplo, que quantifiquem e qualifiquem os estoques de recursos, identifiquem a demanda comunitária e fundamentem técnicas de produção florestal sustentável.

A certificação florestal e o manejo florestal comunitário são reconhecidos como estratégias claras para o manejo florestal sustentável nas florestas tropicais (BURIVALOVA et al., 2017). De modo geral, na região amazônica, a certificação ainda apresenta dificuldades socioambientais e técnicas (SILVA et al., 2016), assim como o manejo florestal comunitário necessita de suporte externo para o desenvolvimento e implementação de práticas de autogestão sustentável (MEDINA; POKORNY; CAMPBELL, 2009). Da mesma forma, as práticas de colheita 
de impacto reduzido são fundamentais para alcançar a sustentabilidade do manejo florestal (PUTZ et al., 2008), assim como as parcerias com multissetores (ROS-TONEM et al., 2008).

Sabe-se que as intervenções planejadas em florestas naturais devem ser precedidas de inventário florestal detalhado, que forneça estimativas confiáveis sobre diversidade, frequência, densidade, dominância e as distribuições diamétrica e espacial das espécies. A partir desses estudos pode-se identificar espécies potenciais ou ainda vulneráveis no manejo e exploração florestal. Para Souza et al. (2006), o conhecimento da composição florística e da estrutura da floresta permite planejar sistemas de manejo com produção sustentável, de modo a conduzir a floresta a uma estrutura balanceada, quando há uma distribuição diamétrica semelhante à original em florestas inequiâneas, com o padrão de J-invertido. Visando subsidiar a implantação de práticas de manejo florestal sustentável comunitário na FLONA do Purus, este estudo teve como objetivo analisar a composição florística, a estrutura florestal e o potencial de manejo florestal de uma área de Floresta Ombrófila Densa de terra firme, que compõe a primeira Unidade de Manejo Florestal da FLONA do Purus.

\section{Material e métodos}

A área de estudo compreende a Unidade de Manejo Florestal-1 (UMF-1) da comunidade Vila Céu do Mapiá, Floresta Nacional do Purus, Pauiní - AM e localiza-se entre as coordenadas UTM657760 E, 9085420 N e 661380 E e 9079879 N, zona 19 L, datum SAD 69 (Figura 1). Segundo Alvares et al. (2013), a classificação de Köppen da região é do tipo Af-tropical equatorial, sem uma estação seca, com temperatura média anual de $24^{\circ} \mathrm{C}$ a $26^{\circ} \mathrm{C}$ e precipitação pluviométrica total anual variando de 2.500 a $2.800 \mathrm{~mm}$. O período com maior intensidade de chuvas compreende os meses de outubro a abril, enquanto o período menos chuvoso, os meses de junho a agosto (IBGE, 1990). Predomina na área a Floresta Ombrófila Densa, com árvores perenifólias sobre Latossolos e Argissolos (BRANDÃO et al., 2010).

Para coleta de dados de vegetação, foram alocadas 12 parcelas de $2.500 \mathrm{~m}^{2}(50 \times 50 \mathrm{~m}) \mathrm{na}$ UMF-1 distribuídas de modo aleatório, totalizando 3,0 hectares de área amostrada (Figura 1). Todas as árvores com diâmetro a altura do peito (dap), definido a $1,30 \mathrm{~m}$ do solo, $\geq 10 \mathrm{~cm}$ foram mensuradas. Para distribuição das espécies em táxons, foi adotado o sistema de classificação da Angiosperm Phylogeny Group - APG III (2009). As espécies amostradas tiveram seu material botânico coletado e encaminhado ao herbário do Jardim Botânico do Rio de Janeiro (JBRJ) para identificação taxonômica, com auxílio de especialistas. As formas de utilização das espécies foram obtidas por meio de entrevistas junto a moradores da Vila Céu do Mapiá, definidas como comerciais as espécies com uso madeireiro. Os dados de campo foram digitados para um banco de dados no Excel e processados no software Mata Nativa 3.11 (CIENTEC, 2011). Foi realizada a análise da composição florística e calculados os índices de Shannon-Wiener ( $\left.\mathrm{H}^{\prime}\right)$ e de Pielou (J) (SHANNON, 1948; PIELOU, 1966). A análise da estrutura florestal foi baseada na estimativa dos parâmetros de densidade, dominância, frequência e valor de importância (MUELLER-DOMBOIS; ELLENBERG, 1974). Para análise do potencial de manejo florestal, além da estrutura da floresta (fitossociologia), foram calculadas a distribuição diamétrica, a distribuição da área basal e as estimativas volumétricas do povoamento (FREITAS; MAGALHÃES, 2012). As estimativas do volume comercial individual da árvore em pé foram obtidas mediante o emprego da seguinte equação (FUNDAÇÃO DE TECNOLOGIA DO ESTADO DO ACRE, 1989): V=0,000308 * $\mathrm{D}^{2,1988}$ , em que: $\mathrm{V}=$ volume comercial individual da árvore em pé, em $\mathrm{m}^{3}$; e $\mathrm{D}=$ diâmetro a altura do peito $(1,30 \mathrm{~m})$, em $\mathrm{cm}$. 


\section{Figura 1 - Localização das parcelas e a Unidade de Manejo Florestal-1 (UMF-1) da Vila Céu do Mapiá, Floresta Nacional do Purus, Pauiní-AM}

Figure 1 - Location of the parcels and the Forest Management Unit (UMF-1) of the Céu do Mapiá village, Purus National Forest, municipality of Pauiní, Amazonas state, Brazil

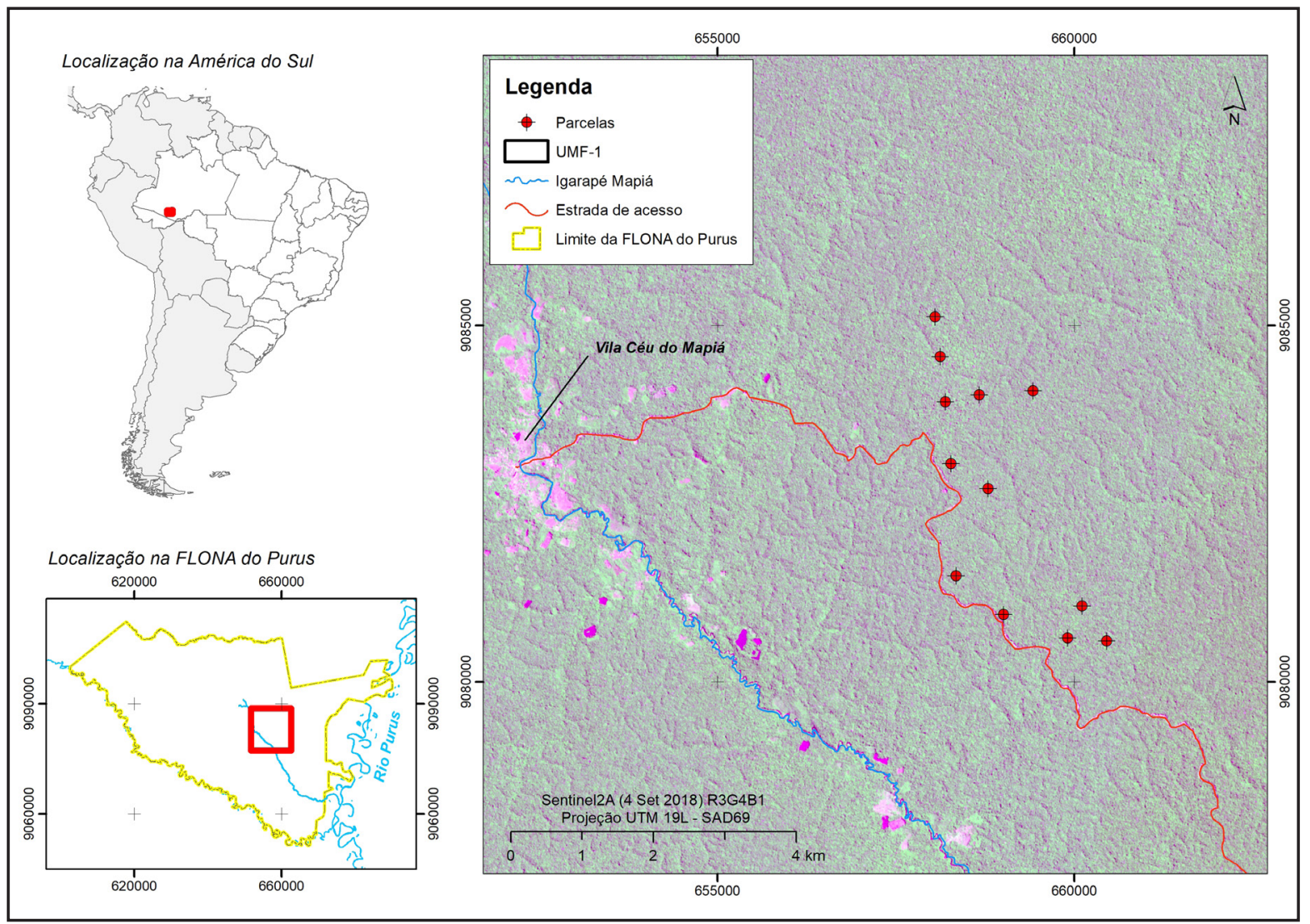

Fonte: Autores (2020)

\section{Resultados e discussão}

Na UMF-1 foram amostrados 1.764 indivíduos (588 indivíduos/ha), representados por 36 famílias botânicas, 91 gêneros e 163 espécimes, das quais 65 foram identificadas em nível de espécie, 74 até o nível de gênero, 15 até o nível de família e 10 indeterminadas (Tabela 1). Fabaceae foi a família com maior riqueza de espécimes (28), seguida de Euphorbiaceae (16), Sapotaceae (13), Lecythidaceae (10), Moraceae (9), Lauraceae (8), Annonaceae (7) e Rubiaceae (6). Sete famílias botânicas contribuíram com um total de 434 (74\%) indivíduos por hectare, evidenciando que poucas famílias botânicas detêm a maior dominância florestal, são elas: Lecythidaceae (306), Euphorbiaceae (249), Sapotaceae (223), Chrysobalanaceae (164), Fabaceae (134), Burseraceae (121) e Moraceae (105). Esses resultados são semelhantes aos encontrados por Barros, Barros e Silva (2000), Maciel, Queiroz e Oliveira (2000), Yared, Couto e Leite (2000), Lima Filho et al. (2001) e Steege et al. (2013), para florestas naturais tropicais de terra firme na Amazônia.

Eschweilera foi o gênero com maior riqueza florística (oito espécies) e com maior número de indivíduos amostrados (299), contribuindo com aproximadamente $17 \%$ do total de árvores mensuradas na amostragem. Resultado semelhante foi encontrado por Steege et al. (2013), ao compilarem e analisarem dados de 1.170 inventários florestais representativos de todas as regiões 
da floresta amazônica. No referido estudo, Eschweilera figura como o gênero mais abundante, representando $5,2 \%$ de todos os indivíduos mensurados.

$\mathrm{O}$ valor de $\mathrm{H}^{\prime}$ obtido de todas as parcelas do universo amostral no presente trabalho foi de 4,10, o que indica uma alta diversidade segundo Knight (1975), sendo o valor de H' obtido na média das parcelas de 3,64. Índices $\mathrm{H}^{\prime}$ estimados em outros trabalhos realizados em florestas naturais tropicais de terra firme da Amazônia também resultaram em valores próximos aos obtidos para a UMF-1 (OLIVEIRA; AMARAL, 2004; ESPIRITO-SANTO et al., 2005; PEREIRA et al., 2005; OLIVEIRA et al., 2008; STEEGE et al., 2013). Quanto ao índice de equabilidade de Pielou (J), o valor encontrado foi de 0,81 , o que corrobora a heterogeneidade da UMF-1 do ponto de vista florístico. As dez espécies com os maiores valores de importância na UMF-1 foram Eschweilerasp.2, Licania brittoniana, Protium sp.1, Pseudolmedia laevigata, Pouteria torta, Eschweilera coriacea, Eschweilera albiflora, Mabeapiriri, Iryanthera juruensis e Micropholis sp.1 que juntas representaram 29\% do valor de importância (Tabela 1). Dentre estas, Licania brittoniana, Pseudolmedia laevigata e Pouteria torta são espécies comerciais madeireiras utilizadas na comunidade e de grande potencial no manejo florestal comunitário.

Doze espécies apresentaram maior densidade, sendo responsáveis por praticamente metade $(48 \%)$ dos indivíduos amostrados, das quais apenas 4 apresentam uso comercial. Dentre estas, as espécies Licania brittoniana e Pseudolmedia laevigata apresentaram frequência absoluta (FA) variando de 91 a 100\% (Tabela 1). A espécie Licania lata também é relatada com fins de artesanato (ALVINO; SILVA; RAYOL, 2005). Além desses, outros usos como fins energéticos (lenha) ou fármacos também poderiam ser considerados para o manejo sustentável das demais espécies não madeireiras (TRINDADE; SILVA; SETZER, 2018; REIS et al., 2019).

As dez espécies com maior dominância absoluta foram Licania brittoniana, Eschweilerasp.2, Protiumsp.1, Pseudolmedia laevigata, Goupia glabra, Micropholis sp.1, Pouteria torta, Caryocar glabrum, Brosimum rubescens e Iryanthera sp.1, que juntas representam $32 \%$ da dominância total (Tabela 1), dentre elas apenas, Eschweilera sp.2, Protiumsp.1 e Micropholis sp.1 não são comerciais. Vale ressaltar que a espécie Goupia glabra contempla apenas um indivíduo, cujo porte permitiulhe figurar entre as dez espécies de maior dominância.

\section{Tabela 1 - Parâmetros fitossociológicos das espécies arbóreas (comerciais e não comerciais) amostradas na FLONA do Purus, município de Pauiní, Estado do Amazonas, Brasil, e classificadas conforme maior Índice de Valor de Importância (VI)}

Table 1 - Phytosociologic parameters of the arborous species (commercial and noncommercial) sampled in Purus National Forest, municipality of Pauiní, Amazonas state, Brazil, and classified by Important Value Index (VI)

\begin{tabular}{|c|c|c|c|c|c|c|c|}
\hline Nome Científico & Família & $\mathbf{N}$ & DA & FA & DoA & VI & Uso \\
\hline Eschweilera sp. 2 & Lecythidaceae & 152 & 50,67 & 100,00 & 1,20 & 14,847 & $\mathrm{NC}$ \\
\hline Licania brittoniana Fritsch & Chrysobalanaceae & 119 & 39,67 & 100,00 & 1,33 & 13,470 & $\mathrm{C}$ \\
\hline Protium sp. 1 & Burseraceae & 115 & 38,33 & 100,00 & 1,15 & 12,577 & $\mathrm{NC}$ \\
\hline Pseudolmedia laevigata Trécul & Moraceae & 63 & 21,00 & 91,67 & 0,82 & 8,259 & C \\
\hline Pouteria torta (Mart.) Radlk. & Sapotaceae & 57 & 19,00 & 75,00 & 0,71 & 7,222 & $\mathrm{C}$ \\
\hline Eschweilera coriacea (DC.) S. A. Mori & Lecythidaceae & 49 & 16,33 & 100,00 & 0,58 & 6,718 & $\mathrm{NC}$ \\
\hline Eschweilera albiflora (DC.) Miers & Lecythidaceae & 54 & 18,00 & 100,00 & 0,49 & 6,674 & $\mathrm{NC}$ \\
\hline Mabeapiriri Aubl. & Euphorbiaceae & 62 & 20,67 & 83,33 & 0,31 & 6,166 & $\mathrm{NC}$ \\
\hline Iryanthera juruensis Warb. & Myristicaceae & 44 & 14,67 & 91,67 & 0,40 & 5,621 & $\mathrm{NC}$ \\
\hline
\end{tabular}


Tabela 1 - Continuação ...

Table 1 - Continuation ...

\begin{tabular}{|c|c|c|c|c|c|c|c|}
\hline Nome Científico & Família & $\mathbf{N}$ & DA & FA & DoA & VI & Uso \\
\hline Micropholis sp. 1 & Sapotaceae & 27 & 9,00 & 66,67 & 0,75 & 5,503 & NC \\
\hline Chrysophyllum amazonicum T. D. Penn. & Sapotaceae & 30 & 10,00 & 91,67 & 0,56 & 5,438 & $\mathrm{P}$ \\
\hline Senefeldera sp. 1 & Euphorbiaceae & 47 & 15,67 & 83,33 & 0,31 & 5,323 & NC \\
\hline Senefeldera macrophylla Ducke & Euphorbiaceae & 55 & 18,33 & 58,33 & 0,27 & 5,156 & NC \\
\hline Pouteria gardneri (Mart. \& Miq.) Baehni & Sapotaceae & 29 & 9,67 & 75,00 & 0,58 & 5,149 & $P$ \\
\hline Brosimum rubescens Taub. & Moraceae & 21 & 7,00 & 83,33 & 0,64 & 5,053 & $\mathrm{C}$ \\
\hline Eschweilera sp. 5 & Lecythidaceae & 25 & 8,33 & 91,67 & 0,53 & 5,037 & $\mathrm{C}$ \\
\hline Ocoteaaciphylla (Nees \& Mart.) Mez & Lauraceae & 28 & 9,33 & 83,33 & 0,50 & 4,945 & $\mathrm{C}$ \\
\hline $\begin{array}{l}\text { Stryphnodendron occhionianum E. M. O. } \\
\text { Martins }\end{array}$ & Fabaceae & 25 & 8,33 & 75,00 & 0,56 & 4,837 & $\mathrm{P}$ \\
\hline Qualea sp. 1 & Vochysiaceae & 31 & 10,33 & 83,33 & 0,42 & 4,806 & $\mathrm{C}$ \\
\hline Glycydendron amazonicum Ducke & Euphorbiaceae & 23 & 7,67 & 75,00 & 0,48 & 4,437 & $\mathrm{C}$ \\
\hline $\begin{array}{l}\text { Zygia ramiflora (Benth.) Barneby \& J. W. } \\
\text { Grimes }\end{array}$ & Fabaceae & 20 & 6,67 & 83,33 & 0,47 & 4,392 & NC \\
\hline Licania sp. 3 & Chrysobalanaceae & 29 & 9,67 & 83,33 & 0,31 & 4,302 & $P$ \\
\hline Hieronyma alchorneoides Allemão & Phyllanthaceae & 29 & 9,67 & 83,33 & 0,26 & 4,122 & $\mathrm{P}$ \\
\hline Iryanthera sp. 1 & Myristicaceae & 10 & 3,33 & 58,33 & 0,64 & 3,964 & $P$ \\
\hline Micropholis egensis (A. DC.) Pierre & Sapotaceae & 30 & 10,00 & 66,67 & 0,23 & 3,773 & $\mathrm{C}$ \\
\hline Hevea sp. 1 & Euphorbiaceae & 16 & 5,33 & 75,00 & 0,39 & 3,686 & NC \\
\hline Caryocar glabrum (Aubl.) Pers. & Caryocaraceae & 7 & 2,33 & 41,67 & 0,68 & 3,657 & $\mathrm{C}$ \\
\hline Micropholis sp. 2 & Sapotaceae & 14 & 4,67 & 58,33 & 0,46 & 3,535 & $\mathrm{C}$ \\
\hline Inga pruriens Poepp. & Fabaceae & 16 & 5,33 & 66,67 & 0,37 & 3,476 & NC \\
\hline Carpotroche sp. 1 & Achariaceae & 12 & 4,00 & 83,33 & 0,30 & 3,290 & NC \\
\hline Casearia arborea (Rich.) Urb. & Salicaceae & 9 & 3,00 & 50,00 & 0,50 & 3,241 & NC \\
\hline Guarea sp. 1 & Meliaceae & 10 & 3,33 & 75,00 & 0,34 & 3,180 & $\mathrm{C}$ \\
\hline Goupia glabra Aubl. & Goupiaceae & 1 & 0,33 & 8,33 & 0,79 & 3,098 & $\mathrm{C}$ \\
\hline Pourouma tomentosa Mart. ex Miq. & Urticaceae & 19 & 6,33 & 75,00 & 0,18 & 3,087 & NC \\
\hline Parkia nitida Miq. & Fabaceae & 8 & 2,67 & 58,33 & 0,41 & 3,011 & $\mathrm{C}$ \\
\hline $\begin{array}{l}\text { Micropholis venulosa (Mart. \& Eichler) } \\
\text { Pierre }\end{array}$ & Sapotaceae & 18 & 6,00 & 58,33 & 0,23 & 2,934 & $\mathrm{C}$ \\
\hline Guatteria sp. 1 & Annonaceae & 12 & 4,00 & 75,00 & 0,23 & 2,900 & NC \\
\hline Tetrorchidium rubrivenium Poepp. & Euphorbiaceae & 14 & 4,67 & 58,33 & 0,28 & 2,891 & $P$ \\
\hline Virola sebifera Aubl. & Myristicaceae & 10 & 3,33 & 75,00 & 0,22 & 2,753 & $P$ \\
\hline Theobroma subincanum Mart. & Malvaceae & 20 & 6,67 & 66,67 & 0,10 & 2,705 & NC \\
\hline Clarisia racemosa Ruiz \&Pav. & Moraceae & 9 & 3,00 & 58,33 & 0,26 & 2,534 & $\mathrm{C}$ \\
\hline Alchornea latifolia Sw. & Euphorbiaceae & 14 & 4,67 & 66,67 & 0,14 & 2,516 & $\mathrm{P}$ \\
\hline & & & & & \multicolumn{3}{|c|}{$\begin{array}{l}\text { Continua ... } \\
\text { Continuatio }\end{array}$} \\
\hline
\end{tabular}


Tabela 1 - Continuação ...

Table 1 - Continuation ...

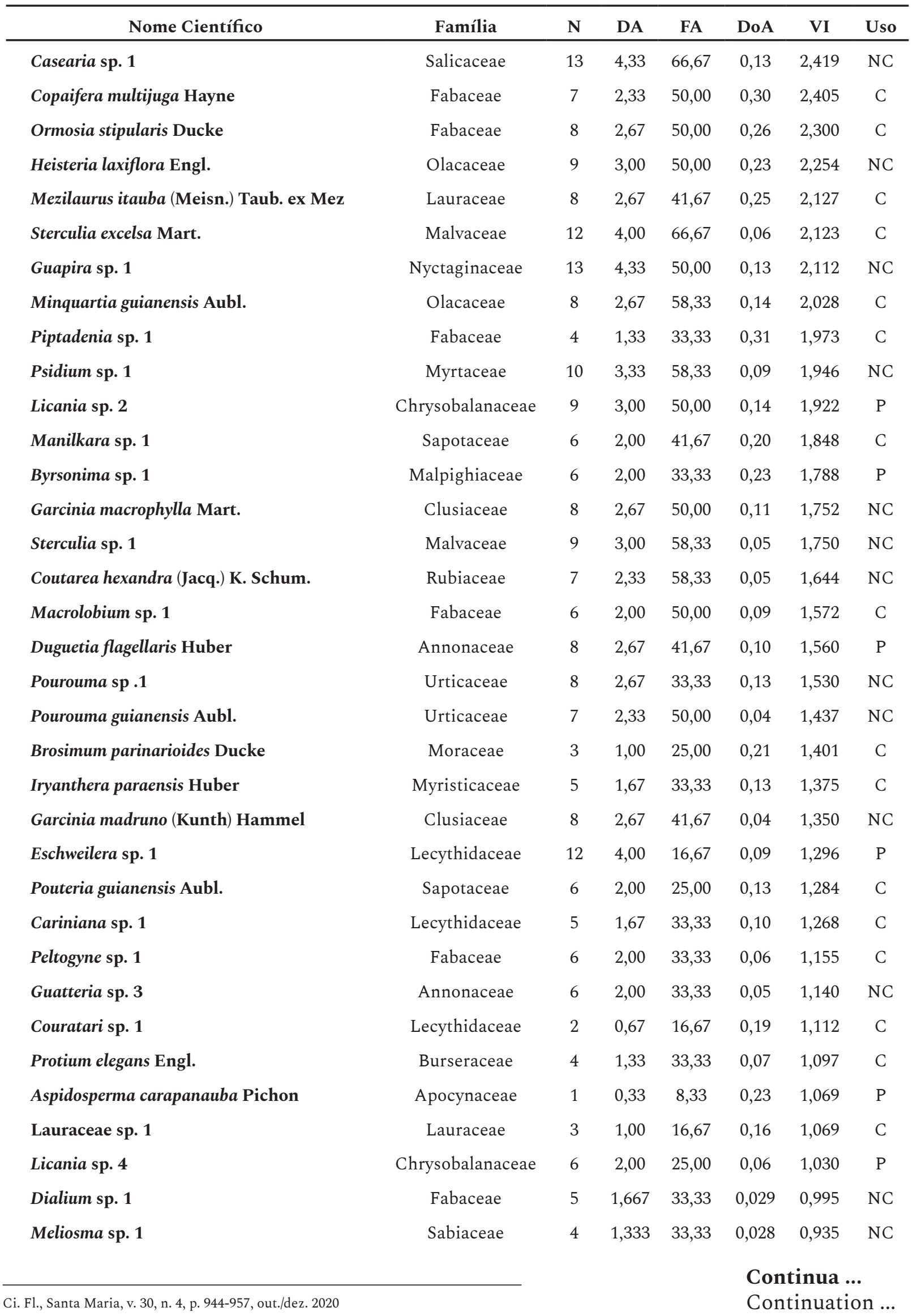


Tabela 1 - Continuação ...

Table 1 - Continuation ...

\begin{tabular}{|c|c|c|c|c|c|c|c|}
\hline Nome Científico & Família & $\mathbf{N}$ & DA & FA & DoA & VI & Uso \\
\hline Myroxylon peruiferum L.f. & Fabaceae & 4 & 1,333 & 33,33 & 0,024 & 0,920 & $\mathrm{C}$ \\
\hline Indeterminada 7 & Indeterminada & 2 & 0,667 & 16,67 & 0,132 & 0,902 & NC \\
\hline Euphorbiaceae 2 & Euphorbiaceae & 3 & 1 & 16,67 & 0,105 & 0,859 & $\mathrm{C}$ \\
\hline Copaifera sp. 1 & Fabaceae & 3 & 1 & 25 & 0,059 & 0,841 & NC \\
\hline Peltogyne catingae Ducke & Fabaceae & 2 & 0,667 & 16,67 & 0,114 & 0,836 & $\mathrm{NC}$ \\
\hline Dipteryx odorata (Aubl.) Willd. & Fabaceae & 3 & 1 & 25 & 0,034 & 0,749 & $\mathrm{C}$ \\
\hline Eschweilera sp. 3 & Lecythidaceae & 4 & 1,333 & 16,67 & 0,059 & 0,747 & NC \\
\hline Mabea sp. 1 & Euphorbiaceae & 6 & 2 & 16,67 & 0,027 & 0,742 & $\mathrm{NC}$ \\
\hline Indeterminada 3 & Indeterminada & 1 & 0,333 & 8,33 & 0,14 & 0,723 & $\mathrm{C}$ \\
\hline Pradosia sp. 1 & Sapotaceae & 3 & 1 & 25 & 0,019 & 0,694 & NC \\
\hline Rubiaceae 1 & Rubiaceae & 3 & 1 & 25 & 0,018 & 0,690 & $\mathrm{NC}$ \\
\hline Psidiumsp.2 & Myrtaceae & 3 & 1 & 25 & 0,012 & 0,668 & $\mathrm{NC}$ \\
\hline Aspidospermasp.1 & Apocynaceae & 1 & 0,333 & 8,33 & 0,124 & 0,664 & $\mathrm{C}$ \\
\hline Indeterminada 6 & Indeterminada & 2 & 0,667 & 16,67 & 0,064 & 0,652 & $\mathrm{NC}$ \\
\hline Erythroxylum sp. 1 & Erythroxylaceae & 2 & 0,667 & 16,67 & 0,059 & 0,633 & C \\
\hline $\begin{array}{l}\text { Helicostylis tomentosa (Poepp. \& Endl.) } \\
\text { Rusby }\end{array}$ & Moraceae & 4 & 1,333 & 16,67 & 0,021 & 0,607 & $\mathrm{C}$ \\
\hline Endlicheria formosa A. C. Sm. & Lauraceae & 3 & 1 & 16,67 & 0,036 & 0,605 & $\mathrm{C}$ \\
\hline Indeterminada 8 & Indeterminada & 3 & 1 & 16,67 & 0,026 & 0,568 & NC \\
\hline Euphorbiaceae 1 & Euphorbiaceae & 3 & 1 & 16,67 & 0,024 & 0,561 & C \\
\hline Indeterminada 9 & Indeterminada & 3 & 1 & 16,67 & 0,018 & 0,539 & NC \\
\hline Pseudoxandra sp. 1 & Annonaceae & 3 & 1 & 16,67 & 0,018 & 0,539 & NC \\
\hline Theobroma microcarpum Mart. & Malvaceae & 2 & 0,667 & 16,67 & 0,016 & 0,475 & $\mathrm{NC}$ \\
\hline Macrolobium sp. 2 & Fabaceae & 2 & 0,667 & 16,67 & 0,015 & 0,471 & $\mathrm{C}$ \\
\hline Stryphnodendron sp. 3 & Fabaceae & 2 & 0,667 & 16,67 & 0,012 & 0,460 & NC \\
\hline Tabebuia sp. 1 & Bignoniaceae & 2 & 0,667 & 16,67 & 0,01 & 0,453 & C \\
\hline Sacoglottis sp. 1 & Humiriaceae & 1 & 0,333 & 8,33 & 0,065 & 0,447 & $\mathrm{NC}$ \\
\hline Protium sp. 2 & Burseraceae & 2 & 0,667 & 8,33 & 0,049 & 0,445 & C \\
\hline Guatteria sp. 2 & Annonaceae & 2 & 0,667 & 16,67 & 0,006 & 0,438 & NC \\
\hline Diplotropis purpurea (Rich.) Amshoff & Fabaceae & 1 & 0,333 & 8,33 & 0,052 & 0,399 & $\mathrm{C}$ \\
\hline Piptadenia sp. 2 & Fabaceae & 1 & 0,333 & 8,33 & 0,05 & 0,392 & C \\
\hline Simarouba amara Aubl. & Simaroubaceae & 2 & 0,667 & 8,33 & 0,033 & 0,386 & $\mathrm{C}$ \\
\hline Indeterminada 5 & Indeterminada & 1 & 0,333 & 8,33 & 0,047 & 0,381 & NC \\
\hline Verbenaceae 1 & Verbenaceae & 3 & 1 & 8,33 & 0,015 & 0,377 & NC \\
\hline Stryphnodendron sp. 1 & Fabaceae & 1 & 0,333 & 8,33 & 0,046 & 0,377 & $\mathrm{NC}$ \\
\hline
\end{tabular}


Tabela 1 - Continuação ...

Table 1 - Continuation ...

\begin{tabular}{|c|c|c|c|c|c|c|c|}
\hline Nome Científico & Família & $\mathbf{N}$ & DA & FA & DoA & VI & Uso \\
\hline $\begin{array}{l}\text { Hevea brasiliensis (Willd. ex A. Juss.) Müll. } \\
\text { Arg. }\end{array}$ & Euphorbiaceae & 1 & 0,333 & 8,33 & 0,041 & 0,359 & NC \\
\hline Dialium sp. 2 & Fabaceae & 1 & 0,333 & 8,33 & 0,041 & 0,359 & NC \\
\hline Duguetia stelechantha (Diels) R. E. Fr. & Annonaceae & 2 & 0,667 & 8,33 & 0,02 & 0,338 & C \\
\hline Platymiscium sp. 1 & Fabaceae & 1 & 0,333 & 8,33 & 0,033 & 0,329 & $\mathrm{C}$ \\
\hline Eschweilera sp. 4 & Lecythidaceae & 2 & 0,667 & 8,33 & 0,018 & 0,331 & NC \\
\hline Amphiodon effusus Huber & Fabaceae & 2 & 0,667 & 8,33 & 0,017 & 0,327 & NC \\
\hline Micropholis sp. 3 & Sapotaceae & 1 & 0,333 & 8,33 & 0,029 & 0,315 & $\mathrm{NC}$ \\
\hline Indeterminada 2 & Indeterminada & 1 & 0,333 & 8,33 & 0,028 & 0,311 & $\mathrm{NC}$ \\
\hline Indeterminada 1 & Indeterminada & 1 & 0,333 & 8,33 & 0,027 & 0,307 & $\mathrm{NC}$ \\
\hline Naucleopsis macrophylla Miq. & Moraceae & 2 & 0,667 & 8,33 & 0,011 & 0,305 & C \\
\hline Aniba canelilla (Kunth) Mez & Lauraceae & 1 & 0,333 & 8,33 & 0,025 & 0,300 & $\mathrm{C}$ \\
\hline Moraceae 1 & Moraceae & 1 & 0,333 & 8,33 & 0,02 & 0,282 & C \\
\hline Nealchornea sp.1 & Euphorbiaceae & 1 & 0,333 & 8,33 & 0,019 & 0,278 & $\mathrm{NC}$ \\
\hline Hymenaea sp. 1 & Fabaceae & 1 & 0,333 & 8,33 & 0,019 & 0,278 & $\mathrm{C}$ \\
\hline Licania sp. 1 & Chrysobalanaceae & 1 & 0,333 & 8,33 & 0,018 & 0,274 & C \\
\hline $\begin{array}{l}\text { Anisophyllea manausensis Pires \& W. A. } \\
\text { Rodrigues }\end{array}$ & Anisophylleaceae & 1 & 0,333 & 8,33 & 0,018 & 0,274 & $\mathrm{NC}$ \\
\hline Indeterminada 4 & Indeterminada & 1 & 0,333 & 8,33 & 0,013 & 0,256 & $\mathrm{NC}$ \\
\hline Miconia sp. 1 & Melastomataceae & 1 & 0,333 & 8,33 & 0,012 & 0,252 & $\mathrm{NC}$ \\
\hline Stryphnodendron sp. 2 & Fabaceae & 1 & 0,333 & 8,33 & 0,011 & 0,248 & $\mathrm{NC}$ \\
\hline Myristicaceae 2 & Myristicaceae & 1 & 0,333 & 8,33 & 0,011 & 0,248 & $\mathrm{C}$ \\
\hline Rubiaceae 2 & Rubiaceae & 1 & 0,333 & 8,33 & 0,011 & 0,248 & $\mathrm{NC}$ \\
\hline Mezilaurus sp. 1 & Lauraceae & 1 & 0,333 & 8,33 & 0,01 & 0,245 & $\mathrm{C}$ \\
\hline Indeterminada 10 & Indeterminada & 1 & 0,333 & 8,33 & 0,01 & 0,245 & $\mathrm{NC}$ \\
\hline Brosimum sp. 1 & Moraceae & 1 & 0,333 & 8,33 & 0,01 & 0,245 & $\mathrm{NC}$ \\
\hline Cecropia sp. 1 & Urticaceae & 1 & 0,333 & 8,33 & 0,01 & 0,245 & NC \\
\hline Annonaceae 1 & Annonaceae & 1 & 0,333 & 8,33 & 0,01 & 0,245 & $\mathrm{NC}$ \\
\hline Nealchornea sp.2 & Euphorbiaceae & 1 & 0,333 & 8,33 & 0,009 & 0,241 & $\mathrm{C}$ \\
\hline $\begin{array}{l}\text { Didymopanax morototoni (Aubl.) Decne. \& } \\
\text { Planch. }\end{array}$ & Araliaceae & 1 & 0,333 & 8,33 & 0,008 & 0,237 & C \\
\hline Alchornea sp. 2 & Euphorbiaceae & 1 & 0,333 & 8,33 & 0,008 & 0,237 & $\mathrm{NC}$ \\
\hline Eriotheca sp. 1 & Malvaceae & 1 & 0,333 & 8,33 & 0,008 & 0,237 & NC \\
\hline Chrysophyllum sp. 2 & Sapotaceae & 1 & 0,333 & 8,33 & 0,008 & 0,237 & $\mathrm{C}$ \\
\hline Euphorbiaceae 3 & Euphorbiaceae & 1 & 0,333 & 8,33 & 0,008 & 0,237 & $\mathrm{NC}$ \\
\hline Eschweilera sp. 6 & Lecythidaceae & 1 & 0,333 & 8,33 & 0,008 & 0,237 & NC \\
\hline & & & & & \multicolumn{3}{|c|}{$\begin{array}{l}\text { Continua .. } \\
\text { Continuatic }\end{array}$} \\
\hline
\end{tabular}


Tabela 1 - Conclusão ...

Table 1 - Conclusion ...

\begin{tabular}{|c|c|c|c|c|c|c|c|}
\hline Nome Científico & Família & $\mathbf{N}$ & DA & FA & DoA & VI & Uso \\
\hline Senefeldera sp. 2 & Euphorbiaceae & 1 & 0,333 & 8,33 & 0,007 & 0,234 & $\mathrm{NC}$ \\
\hline Simarouba sp. 1 & Simaroubaceae & 1 & 0,333 & 8,33 & 0,007 & 0,234 & $\mathrm{C}$ \\
\hline Pourouma sp. 2 & Urticaceae & 1 & 0,333 & 8,33 & 0,007 & 0,234 & $\mathrm{NC}$ \\
\hline Moraceae 2 & Moraceae & 1 & 0,333 & 8,33 & 0,007 & 0,234 & $\mathrm{C}$ \\
\hline Fabaceae 2 & Fabaceae & 1 & 0,333 & 8,33 & 0,006 & 0,230 & $\mathrm{NC}$ \\
\hline Miconia sp. 2 & Melastomataceae & 1 & 0,333 & 8,33 & 0,006 & 0,230 & $\mathrm{NC}$ \\
\hline Carapa guianensis Aubl. & Meliaceae & 1 & 0,333 & 8,33 & 0,006 & 0,230 & $\mathrm{C}$ \\
\hline Chrysophyllum sp. 3 & Sapotaceae & 1 & 0,333 & 8,33 & 0,005 & 0,226 & $\mathrm{NC}$ \\
\hline $\begin{array}{l}\text { Pseudopiptadenia psilostachya (DC.) G. P. } \\
\text { Lewis \& M. P. Lima }\end{array}$ & Fabaceae & 1 & 0,333 & 8,33 & 0,005 & 0,226 & $\mathrm{NC}$ \\
\hline Fabaceae 1 & Fabaceae & 1 & 0,333 & 8,33 & 0,005 & 0,226 & NC \\
\hline Simira sp. 1 & Rubiaceae & 1 & 0,333 & 8,33 & 0,004 & 0,223 & NC \\
\hline Garcinia sp. 2 & Clusiaceae & 1 & 0,333 & 8,33 & 0,004 & 0,223 & NC \\
\hline Apuleia sp. 1 & Fabaceae & 1 & 0,333 & 8,33 & 0,004 & 0,223 & $\mathrm{C}$ \\
\hline Lauraceae 3 & Lauraceae & 1 & 0,333 & 8,33 & 0,004 & 0,223 & $\mathrm{C}$ \\
\hline Lauraceae 2 & Lauraceae & 1 & 0,333 & 8,33 & 0,004 & 0,223 & $\mathrm{C}$ \\
\hline Coussarea macrophylla (Mart.) Müll. Arg & Rubiaceae & 1 & 0,333 & 8,33 & 0,004 & 0,223 & NC \\
\hline Garcinia sp. 1 & Clusiaceae & 1 & 0,333 & 8,33 & 0,004 & 0,223 & $\mathrm{NC}$ \\
\hline Guarea sp. 2 & Meliaceae & 1 & 0,333 & 8,33 & 0,003 & 0,219 & $\mathrm{NC}$ \\
\hline Agouti carpa curviflora (Dwyer) C. H. Perss. & Rubiaceae & 1 & 0,333 & 8,33 & 0,003 & 0,219 & $\mathrm{NC}$ \\
\hline Total & & 1764 & 588 & 5508 & 27,158 & 300 & - \\
\hline
\end{tabular}

Fonte: Autores (2020)

Em que: $\mathrm{N}=$ número de indivíduos; $\mathrm{DA}=$ densidade absoluta $\left(\mathrm{n} \mathrm{ha}^{-1}\right) ; \mathrm{FA}=$ frequência absoluta; DoA = dominância absoluta $\left(\mathrm{m}^{2}\right.$ ha $\left.\mathrm{a}^{-1}\right)$; VI = valor de importância; $\mathrm{NC}=$ não comercial; $\mathrm{C}=$ comercial; $\mathrm{P}$ = potencial uso comercial.

Considerando como espécies de baixa abundância aquelas que tiveram um ou dois indivíduos amostrados, chamadas respectivamente de Unicatas e Duplicatas (FERRAZ; GADELHA; AGUIAR-COELHO, 2009) pode-se inferir que na UMF-1, 45\% (73) das espécies são de baixa abundância, das quais 78\% (57) são Unicatas e 22\% (16) Duplicatas. Das espécies identificadas e de baixa abundância do universo amostral (3 ha), aproximadamente 11\% (8) pertencem ao grupo das espécies comerciais utilizadas localmente e devem ser reservadas como matrizes, são elas: Aniba canelilla, Carapa guianensis, Didymopanax morototoni, Diplotropis purpurea, Duguetia stelechantha, Goupia glabra, Naucleopsis macrophylla e Simarouba amara.

A comunidade florística da UMF-1 apresentou estrutura diamétrica com tendência a J-invertido (Figura 2), que é o padrão característico das florestas inequiâneas (SOUZA et al., 2006). Foram estimados, considerando-se o dap $\geq 10 \mathrm{~cm}, 589$ ind.ha ${ }^{-1}$ na UMF-1, sendo que $66,6 \%$ dos indivíduos apresentaram diâmetro inferior a $20 \mathrm{~cm}$ e inferiores de $50 \mathrm{~cm}$, acumularam, 96,3\% dos indivíduos apresentam dap $50 \mathrm{~cm}$. Ressaltando que apenas 3,7\% dos indivíduos apresentaram diâmetro comercial para o manejo, dap $\geq 50 \mathrm{~cm}$. Os intervalos de classes de $100 \leq \mathrm{dap}<140$ e $150 \leq$ dap $<170$ não apresentaram indivíduos e as classes $140 \leq \mathrm{dap}<150$ e $170 \leq \mathrm{dap}<180$ 
apresentaram, respectivamente, a ocorrência de 0,33 ind.ha ${ }^{-1}$, o que representa a mensuração de apenas um indivíduo em cada classe. O diâmetro máximo encontrado foi de $173,2 \mathrm{~cm}$, referente a um indivíduo de Goupia glabra.

Figura 2 - Densidade absoluta de indivíduos por hectare (DA) por classe de diâmetro para Unidade de Manejo Florestal 1 da Vila Céu do Mapiá, Floresta Nacional do Purus, PauiníAM

Figure 2 - Absolute density of individuals per hectare (DA) by diameter class for the Forest Unit Management of the Céu do Mapiá Village, Purus National Forest, Municipality of Pauiní, Amazon State, Brazil

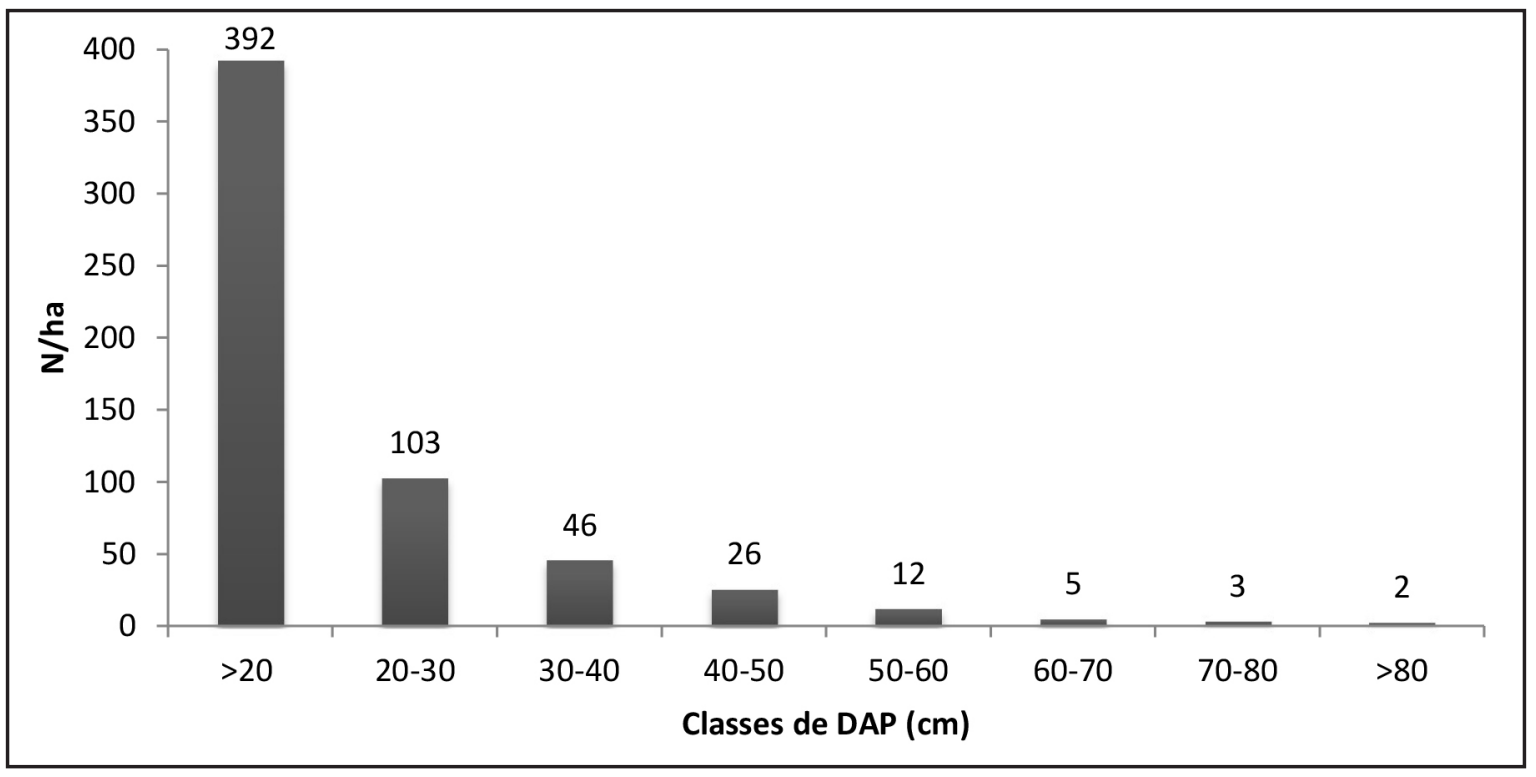

Fonte: Autores (2020)

Do total de 163 espécies arbóreas do inventário florestal amostral da UMF-1, 65 (40\%) foram classificadas como produtoras de madeiras comerciais, ou seja, aquelas que atualmente possuem mercado local definido e 15 (9\%) como espécies comerciais potenciais. Contudo, considerando-se as classes diamétricas na qual é feita a seleção de indivíduos para corte, dap $\geq$ $50 \mathrm{~cm}$ (BRASIL, 2009), ocorrem apenas 26 das espécies comerciais. Esse valor representa $32 \%$ do número de espécies comerciais ou potenciais e 15\% do número total de espécies da UMF-1 e está abaixo da média esperada para a região amazônica, que é de 35 espécies exploradas por plano de manejo (SOUZA, 2005). Esse baixo nível de aproveitamento da totalidade das espécies, em função do padrão de dispersão, é apontado como um dos principais entraves para a exploração econômica da Floresta Amazônica (BRASIL, 1976). Entretanto, esses números ainda podem estar subestimados, pois, segundo a Resolução CONAMA no 406 (BRASIL, 2009), o Diâmetro Mínimo de Corte é estabelecido por espécie manejada, a partir de estudos técnicos envolvendo sua distribuição e características ecológicas, bem como ao seu uso comercial. Além disso, os métodos de amostragem também podem influenciar na acurácia dos resultados (KAUAI et al., 2019), o que reforça a importância do inventário 100\% para otimização do manejo florestal sustentável.

Os indivíduos das espécies comerciais com dap $\geq 50 \mathrm{~cm}$ somam um total de densidade de 22 ind ha ${ }^{-1}$, uma dominância de 7,0 $\mathrm{m}^{2} \mathrm{ha}^{-1}$ e um volume de fuste de $65,6 \mathrm{~m}^{3} \mathrm{ha}^{-1}$. A produtividade média por árvore foi de $2,050 \mathrm{~m}^{3} \mathrm{ind}^{-1} \mathrm{ha}^{-1}$, em valores absolutos de $3,592 \mathrm{~m}^{3} \mathrm{ind}^{-1}$. Ressaltando que esses números absolutos são amostrais, não representativos principalmente devido à alta riqueza de espécies. De modo que, a média amostral de 22 indivíduos detectado por hectare é composta 
por diversas espécies, podendo essas representarem abundância inferior a 3\% na área de manejo, o que pelas normativas de manejo proíbe a sua exploração. Outros resultados são encontrados em áreas florestais do Projeto de Assentamento Agroextrativista (PAE) de Porto Dias (FUNDAÇÃO DE TECNOLOGIA DO ESTADO DO ACRE, 1996) e da Floresta Nacional do Macauã (CENTRO DE TRABALHADORES DA AMAZONIA, 1998), considerando apenas as espécies comerciais nas classes de tamanho indicadas para exploração $\left(40\right.$ ind.ha ${ }^{-1}$ com $81,5 \mathrm{~m}^{3}$ ha $^{1}$ e 33 ind. ${ }^{-1} \mathrm{ha}^{-1} \mathrm{com}$ $51 \mathrm{~m}^{3} \mathrm{ha}^{-1}$, respectivamente).

Das 26 espécies comerciais selecionadas, apenas Goupia glabra não apresentou estoque remanescente, reduzindo para $38 \%$ a estimativa de espécies comerciais com estoque suficiente para o manejo florestal sustentável.

\section{Conclusões}

A estrutura da floresta na UMF-1 caracteriza-se pela alta diversidade florística, poucas espécies são dominantes e muitas espécies de baixa densidade florestal.

Ressaltando que a maior dificuldade para o manejo florestal comunitário é o desconhecimento botânico, com praticamente menos da metade das espécies tendo recebido a identificação botânica completa, o que pode implicar em resultados negativos à sustentabilidade ecológica e econômica do manejo florestal.

A UMF-1 apresenta potencial de manejo para 26 espécies comerciais, uma vez que são espécies de baixa abundância. Entretanto, o inventário 100\% é prioritário para otimizar o manejo florestal sustentável e pode aumentar esse número. É necessária a pesquisa de novas áreas, para se ter uma avaliação mais segura do potencial de manejo dessas espécies na Zona de Uso Comunitário.

O estoque comercial da UMF-1 da Vila Céu do Mapiá permite a produção de madeiras contínua (curto, médio e longo prazo), desde que realizada com base nos princípios do manejo florestal sustentável. Este estudo subsidiou a implantação do Plano de Manejo Florestal Sustentável de Baixa Intensidade da Comunidade Vila Céu do Mapiá, atualmente em fase de colheita das espécies comerciais madeireiras.

\section{Agradecimentos}

Ao CNPq (Conselho Nacional de Desenvolvimento Científico e Tecnológico) pelo apoio financeiro no projeto e à CAPES (Coordenação de Aperfeiçoamento de Pessoal de Nível Superior) pela bolsa de estudos do primeiro autor.

\section{Referências}

ALVARES, C. A. et al. Köppen's climate classification map for Brazil. Meteorologische Zeitschrift, Berlin, v. 22, n. 6, p. 711-728, 2013.

ALVINO, F. de O.; SILVA, M. F. F. da; RAYOL, B. P. Potencial de uso das espécies arbóreas de uma floresta secundária, na Zona Bragantina, Pará, Brasil. ActaAmazonica, Manaus, v. 35, n. 4, p. 413-420, 2005.

ANGIOSPERM PHYLOGENY GROUP. An update of the Angiosperm Phylogeny Group classification for the orders and families of flowering plants: APG III. Journal of the Linnean Society, London, v. 161, n. 2, p. 105-121, 2009.

ARAGÃO, L. E. et al. 21st Century drought-related fires counteract the decline of Amazon deforestation carbon emissions. Nature Communications, [s. l.], v. 9, n. 1, p. 536, 2018.

BARROS, A. V.; BARROS, P. L. C., SILVA, L. C. B. Análise fitossociológica de uma floresta situada em Curuá-Una - Pará. Revista Ciências Agrárias, Recife, n. 34, p. 9-36, 2000. 
BRANDÃO, P. C. et al. Caracterização de geoambientes da floresta nacional do Purus, Amazônia Ocidental: uma contribuição ao plano de manejo. Revista Árvore, Viçosa, MG, v. 34, n. 1, p. 115-126, 2010. DOI:10.1590/ S0100-67622010000100013

BRASIL. Decreto n. 96.190, de 21 de junho de 1988. Cria, no Estado do Amazonas, a Floresta Nacional do Purus, com limites que especifica e dá outras providências. Diário Oficial [da] República Federativa do Brasil, Brasília, DF, 22 de jun. 1988. Disponível em: www4.icmbio.gov.br/flonas/legislacao.php?id_arq=51. Acesso em: 20 jan. 2014.

BRASIL. Ministério das Minas e Energia. Departamento Nacional da Produção Mineral. Projeto RADAMBRASIL. Folha SC. 19, Rio Branco: geologia, geomorfologia, pedologia, vegetação e uso potencial da terra. Rio de Janeiro, 1976. v. 12. 464 p.

BRASIL. Resolução CONAMA n. 406, de 2 de fevereiro de 2009. Estabelece parâmetros técnicos a serem adotados na elaboração, apresentação, avaliação técnica, e execução de Plano de Manejo Florestal Sustentável - PMFS com fins madeireiros, para florestas nativas e suas formas de sucessão no Bioma Amazônia. Brasília, 2009.4 p.

BURIVALOVA, Z. et al. A Critical Comparison of Conventional, Certified, and Community Management of Tropical Forests for Timber in Terms of Environmental, Economic, and Social Variables. Conservation Letters, [s. l.], v. 10, n. 1, p. 4-14, 2016. DOI:10.1111/conl.12244

CENTRO DE TRABALHADORES DA AMAZONIA. Floresta Nacional do Macauã: Inventário Florestal. Rio Branco, 1998. v. 1.

CIENTEC. Software Mata Nativa 3.11: sistema para análise fitossociológica, elaboração de inventários e planos de manejo de florestas nativas. Viçosa, MG, 2011.

ESPÍRITO-SANTO, F. D. B. et al. Análise da composição florística e fitossociologia da Floresta Nacional do Tapajós com apoio geográfico de imagens de satélites. Acta Amazonica, Manaus, v.35, n. 2, p. 155-173, 2005.

FERRAZ, A. C. P.; GADELHA, B. Q.; AGUIAR-COELHO, V. M. Análise faunística de Calliphoridae (Diptera) da Reserva Biológica do Tinguá, Nova Iguaçu, Rio de Janeiro. Revista Brasileira de Entomologia, Curitiba, v. 53, n. 4, p. 620-628, dez. 2009.

FREITAS, W. K. de; MAGALHÃES, L. M. S. Métodos e parâmetros para estudo da vegetação com ênfase no estrato arbóreo. Floresta e Ambiente, Seropédica, v. 19, n. 4, p. 520-540, 2012. DOI: 10.4322/floram.2012.054

FUNDAÇÃO DE TECNOLOGIA DO ESTADO DO ACRE. Estrutura do plano de manejo de uso múltiplo da floresta Estadual do Antimary. Rio Branco, 1989.

FUNDAÇÃO DE TECNOLOGIA DO ESTADO DO ACRE. Floresta Estadual do Antimary. Estudos Básicos. Rio Branco, 1996.v. 1.

IBGE. Diagnóstico geoambiental e sócio-econômico: área de influência da BR-364 trecho Porto Velho/Rio Branco. Rio de Janeiro, 1990. v. 1. 132 p.

INSTITUTO CHICO MENDES DE CONSERVAÇÃO DA BIODIVERSIDADE. Plano de Manejo da Floresta Nacional do Purus. Brasília, DF: IBAMA, 2009. 663 p. Disponível em: http://www.icmbio.gov.br/ . Acesso em: 20 jan. 2014.

INSTITUTO NACIONAL DE PESQUISAS ESPACIAIS (Brasil). Taxas Anuais do Desmatamento na Amazônia Legal - 1988 até 2013. [S. l.], 2013. Disponível em http://www.obt.inpe.br/prodes/ prodes_1988_2013.htm. Acesso em: 10 jan. 2014.

KAUAI, F. et al. Evaluation of forest inventory processes in a forest under concession in the southwestern Brazilian Amazon. ActaAmazonica, Manaus, v. 49, n. 2, p. 91-96, 2019. DOI: 10.1590/1809-4392201801331

KNIGHT, D. H.A phytosociological analysis of species-rich tropical forest on Barro Colorado Island, Panama. Ecological Monographs, Washington, v. 45, p. 259-284, 1975. 
LIMA FILHO, D. A. et al. Inventário florístico de Floresta Ombrófila Densa de terra firme, na região do rio Urucu-Amazonas, Brasil. Acta Amazonica, Manaus, v. 31, n. 4, p. 565-579, 2001.

MACIEL, M. N. M.; QUEIROZ, W. T.; OLIVEIRA, F. A. Parâmetros fitossociológicos de uma floresta tropical de terra firme na Floresta Nacional de Caxiuaunã - PA. Revista Ciências Agrárias, Recife, n. 34, p. 85-106, 2000.

MEDINA, G.; POKORNY, B.; CAMPBELL, B. Community forest management for timber extraction in the Amazon frontier. International Forestry Review, Oxford, v. 11, n. 3, p. 408-420, 2009. DOI: 10.1505/ ifor.11.3.408.

MUELLER-DOMBOIS, D.; ELLENBERG, H. Aims and methods of vegetation ecology. New York: John Wiley \& Sons, 1974. 547 p.

OLIVEIRA, A. N. de et al. Composição e diversidade florístico-estrutural de um hectare de floresta densa de terra firme na Amazônia Central, Amazonas, Brasil. Acta Amazonica, Manaus, v. 38, n. 4, p. 627-642, 2008.

OLIVEIRA, A. N.; AMARAL, I. L. Florística e fitossociologia de uma floresta de vertente na Amazônia Central, Amazonas, Brasil. Acta Amazonica, Manaus, v. 34, p. 21-34, 2004.

PEREIRA, N. W. V. et al. Análise das variações temporais na florística e estrutura da comunidade arbórea de uma floresta explorada com plano de manejo. Revista Cerne, Lavras, v. 11, n. 3, p. 263-282, 2005.

PIELOU, E. C. The measurement of diversity in different types of biological collections. Journal of Theoretical Biology, London, v. 13, p. 131-144, 1966.

PUTZ, F. E. etal. Reduced-impact logging: Challenges and opportunities. Forest Ecologyand Management, Amsterdam, v. 256, n. 7, p. 1427-1433, 2008. DOI: 10.1016/j.foreco.2008.03.036

REIS, J. S. et al. Combustion properties of potential Amazon biomass waste for use as fuel. Journal of Thermal Analysis and Calorimetry, Budapest, v. 138, p. 3535-353, 2019. DOI: 10.1007/s10973-019-08457-5

ROS-TONEN, M. A. F. et al. Forest-related partnerships in Brazilian Amazonia: there is more to sustainable forest management than reduced impact logging. Forest Ecology and Management, Amsterdam, v. 256, n. 7, p. 1482-1497, 2008. DOI: 10.1016/j.foreco.2008.02.044

SHANNON, C. E. A mathematical theory of communication. Bell System Technical Journal, New York, v. 27, p. 379-423, 1948.

SILVA, E. V. da et al. Quais os principais desvios do manejo florestal da Amazônia brasileira perante a certificação? Revista de Ciências Agrárias, Recife, v. 59, n. 4, p. 393-400, 2016.

SONTER, L. L. et al. Mining drives extensive deforestation in the Brazilian Amazon. Nature Communications, [s. l.], v. 8, p. 1013, 2017. DOI: 10.1038/s41467-017-00557-w

SOUZA, D. R. et al. Análise estrutural em Floresta Ombrófila Densa de terra firme não explorada, Amazônia Oriental. Revista Árvore, Viçosa, MG, v. 30, n. 1, p. 75-87, 2006.

SOUZA, R. M. O. Avaliação ecológica rápida para o levantamento do potencial florestal da Floresta Nacional do Purus. Relatório. Rio Branco: [s. n.], 2005. 81 p.

SPRACKLEN, D. V.; GARCIA-CARRERAS, L. The impact of Amazonian deforestation on Amazon basin rainfall. Geophysical Research Letters, Washington, v. 42, p. 9546-9552, 2015. DOI:10.1002/2015GL066063

STEEGE, H. et al. Hyperdominance in the Amazonian Tree Flora. Science, [s. l.], v. 342, p. 6159, 2013.

TRINDADE, R. da; SILVA, J. K. da; SETZER, W. N. Copaifera of the Neotropics: a review of the phytochemistry and pharmacology. International Journal of Molecular Sciences, [s. l.], v. 19, p. 1511, 2018

YARED, J. A. G.; COUTO, L.; LEITE, H. G. Diversidade de espécie em floresta secundária e primária, sob efeito de diferentes sistemas silviculturais, na Amazônia Oriental. Revista Árvore, Viçosa, MG, v. 24, n. 1, p. 83-90, 2000. 